\title{
Apical periodontitis as potential source of infection in patients with lymphoma treated with chemotherapy
}

\author{
Kristina Skallsjö $^{1}$ • Jan-Erik Johansson ${ }^{2,3} \cdot$ Peter Jonasson $^{4} \cdot$ Bengt Hasséus $^{5}$
}

Received: 14 March 2017 / Accepted: 9 April 2019 / Published online: 1 May 2019

(C) The Author(s) 2019

\begin{abstract}
Objectives To investigate signs of infection and infection-related complications of apical periodontitis (AP) in patients who underwent chemotherapy for lymphoma.

Materials and methods Data were collected retrospectively from the dental and medical records of patients receiving chemotherapy for lymphoma. Based on the findings from a dental evaluation made in conjunction with chemotherapy, the patients were divided into two groups, patients with or without teeth with AP.

Results Eighty-six of the 213 patients had one or more teeth with AP and received no planned dental treatment for this condition, while 127 patients had no AP-affected teeth. During chemotherapy, seven patients (8\%) developed local symptoms related to teeth with AP, while no patients in the control group developed symptoms of AP. No significant differences were found with respect to the administration of antibiotics related to dental infection or hospital admission events due to fever or infection, between the group with AP and the group without AP.

Conclusions AP is a common finding and exacerbation seems more common in patients diagnosed with chronic AP than in patients without chronic AP. The presence of chronic AP in patients treated with chemotherapy for lymphoma is not linked to additional medical complications that require hospital admission owing to fever/infection.

Clinical relevance Knowledge regarding infection-related complications of AP in patients with lymphoma treated with chemotherapy will guide clinical decision-making by identifying those patients who warrant treatment. This will allow dental interventions to be postponed until completion of chemotherapy, without serious medical complications. The results of this study serve as a basis for larger prospective studies.
\end{abstract}

Keywords Oral infection $\cdot$ Lymphoma $\cdot$ Apical periodontitis $\cdot$ Exacerbation $\cdot$ Immunosuppression

Bengt Hasséus

Bengt.Hasseus@odontologi.gu.se

1 Clinic of Orofacial Medicine, Mölndal, Public Dental Service, 431 80 Mölndal, Sweden

2 Department of Hematology and Coagulation, Sahlgrenska University Hospital, Gothenburg, Sweden

3 Department of Internal Medicine and Clinical Nutrition, Institute of Medicine, Sahlgrenska Academy, University of Gothenburg, Gothenburg, Sweden

4 Department of Endodontology, Institute of Odontology, The Sahlgrenska Academy, University of Gothenburg, Box 450, 405 30 Gothenburg, Sweden

5 Department of Oral Medicine and Pathology, Institute of Odontology, The Sahlgrenska Academy, University of Gothenburg, Box 450, 40530 Gothenburg, Sweden

\section{Introduction}

Chemotherapy is the first-line treatment for malignant blood diseases, such as leukemia, lymphoma, and myeloma. Immunosuppression to varying degrees is an inevitable consequence of this treatment. The cytotoxic effect of the chemotherapeutic drug on blood cells results in leukopenia to a degree that depends on the type of drug and dosing regimen used [1]. As a consequence of immunosuppression, anatomical sites (such as the oral cavity) that normally harbor microorganisms are potential foci for infection and systemic spread of microorganisms, resulting in septicemia [1].

Treatment of patients with lymphoma typically involves different combinations of chemotherapeutic drugs given in cycles with short intervals (2-4 weeks). Following diagnosis and a decision on the treatment protocol, treatment is initiated immediately. As a result, there is little time or possibility to 
perform a dental examination before the first chemotherapy cycle. As a consequence, patients may enter the treatment with ongoing dental disease.

Apical periodontitis (AP), which is a symptomatic or asymptomatic inflammatory condition of the periradicular tissue of the teeth, results from interactions between microorganisms in an infected root canal system and the host's immune system [2]. AP may develop in response to a primary infection in a necrotic pulp or after root canal treatment due to a persisting infection or a reinfection in inadequately treated teeth. Although the incidence of AP in the healthy population of Sweden in general is slowly decreasing due to improvements in dental health, it remains a common condition, and its prevalence increases with age. The majority of adult Swedes have at least one root-filled tooth. The prevalence of AP in the Swedish population is approximately $30 \%$, and $25 \%$ of the root-filled teeth present signs of persistent infection and inflammation upon radiographic examination [3].

The risk for exacerbation of AP to occur and become symptomatic with local or systemic signs of infection is not well known [4]. Based on repetitive cross-sectional studies of AP in both untreated and root-filled teeth, Eriksen estimated the incidence to be less than 5\% per year [5]. In a group of patients with persistent AP after endodontic treatment, the incidence of exacerbation with local signs of infection was even lower, at less than $6 \%$ over a period of 20 years [6]. Thus, the risk for exacerbation of AP in a healthy population must be considered to be low, albeit not negligible. A recent systematic review calculated the weighted prevalence of dental infections/abscess during chemotherapy to be $5.4 \%$ [7]. In the immunosuppressed patient with neutropenia, all emerging infections can result in a severe systemic infection, requiring hospital admission and intravenous treatment with broad-spectrum antibiotics $[1,8]$. The gold standard for preparing patients for treatment with cytotoxic drugs includes, if possible, a dental clinical and radiographic examination to identify and eliminate dental infection foci before starting chemotherapy [9-11]. For patients with lymphoma, for whom chemotherapy starts almost immediately after establishment of the diagnosis, this option is limited, which means that patients may enter the medical treatment with AP.

The aim of this retrospective study was to investigate infection-related complications of AP in patients who underwent standard dose chemotherapy without stem cell support for lymphoma.

\section{Methods}

The dental and medical records of patients $(N=284)$ who received a diagnosis of lymphoma at the Department of Hematology and Coagulation, Sahlgrenska University Hospital, Gothenburg and who were referred, according to the clinical care protocol, for dental evaluation at the Clinic of Oral Medicine in the period 2005-2013 were consecutively reviewed. The standard dental evaluation included a medical and dental history, including self-reported symptoms, clinical inspection of soft and hard oral tissues, and complete intraoral and panoramic radiographic surveys. A consultant in oromaxillofacial radiology interpreted all the radiographic images. All included lymphoma patients were planned for standard dose chemotherapy without stem cell support.

The time interval between lymphoma diagnosis and start of chemotherapy is usually very short. Therefore, the majority of the study patients had already started chemotherapy when the dental evaluation was performed. The clinical evaluation included inspection of oral tissues; a full assessment of periodontal status by probing is not routinely conducted in our center for patients with cancer who have started chemotherapy.

The inclusion criteria for the study group were (i) a full dental examination, including radiographic survey, and (ii) the presence of at least one tooth with periapical radiolucency. AP was defined as the presence of a periapical radiolucency and absence of lamina dura, judged as periapical destruction by the radiologist, and in not root-filled teeth without response to sensitivity testing. The control group fulfilled criterion (i) but not criterion (ii).

The exclusion criteria were lack of complete record data, missing radiographs, complete edentulousness or a full dentition consisting of implants, and cessation of a planned chemotherapy regimen due to unforeseen medical complications. Fifty patients were excluded based on these criteria.

In the remaining group of 234 patients, 20 received immediate dental treatment upon dental evaluation and were excluded from further analysis, since no teeth with AP remained after the dental treatment. In the excluded group, three patients were scheduled for autologous stem cell transplantation after initial chemotherapy. One patient had a severely deteriorated dental status in combination with severe dental anxiety and received dental treatment under general anesthesia. Nine patients had teeth with AP that were adjudged to be non-treatable due to severe loss of tooth substance, so extractions were performed. Seven patients had teeth with AP for which endodontic treatment was performed during chemotherapy following an individual risk assessment carried out by the dentist and not related to symptoms. In the remaining cohort of 214 patients, one patient had a complex medical situation with repeated systemic infections and was admitted to the hospital for 15 weeks (110 days) during chemotherapy protocol. This patient was considered an outlier and was excluded from the analysis. Thus, the cohort that proceeded to further review consisted of $213 \mathrm{pa}-$ tients, of whom 86 had one or more teeth with AP (study group) (Table 1). The 127 patients without AP formed the control group (Table 1). The follow-up observation period in the medical and dental records was set to day 1 of the first chemotherapy cycle, with the endpoint set at 4 weeks after the start of the last chemotherapy cycle. 
Table 1 Clinical characteristics of patients receiving chemotherapy

Patients with AP

(no. patients/no. cycles)

No.

Age (years)

Sex

Diagnosis

(ICD-10 classification)

Diffuse large B cell lymphon
Mature T/NK cell lymphoma
Other lymphoma (C.85; C.86)
Primary disease
Relapse
ABVD-28
BEACOPP-14
CHOEP-14
CHOP-14
CHOP-21
COP-21
GA101 + Bendamustine-28
Hyper-CVAD-14
ICE-21
MCL2-21
R-Bendamustine-28
R-CHOEP-14
R-CHOP-14
R-CHOP-21
R-COEP-21
R-COP-21
R-CP-21
R-ICE-21
Other

$\begin{array}{ll}86 & 127 \\ 65 ; 66(29-91) & 53 ; 55(20-86) \\ 44(51 \%) & 69(54 \%) \\ 42(49 \%) & 58(46 \%) \\ 5 & 22 \\ 17 & 22 \\ 54 & 68 \\ 4 & 7 \\ 6 & 8 \\ 66 & 94 \\ 20 & 33 \\ 4(2 / 2,2 / 6) & 16(1 / 2,15 / 6) \\ 0 & 3(1 / 6,2 / 8) \\ 1(1 / 6) & 7(7 / 6) \\ 3(1 / 3,2 / 6) & 2(2 / 6) \\ 2(1 / 5,1 / 8) & 2(1 / 3,1 / 6) \\ 3(2 / 6,1 / 8) & 0 \\ 0 & 3(3 / 6) \\ 1(1 / 2) & 3(1 / 5,2 / 8) \\ 1(1 / 3) & 3(2 / 3,1 / 4) \\ 5(5 / 6) & 3(2 / 6,1 / 7) \\ 5(5 / 6) & 6(1 / 1,1 / 5,4 / 6) \\ 2(2 / 6) & 15(1 / 4,1 / 5,12 / 6,1 / 8) \\ 23(22 / 6,1 / 8) & 18(17 / 6,1 / 8) \\ 22(1 / 3,15 / 6,1 / 7,5 / 8) & 25(3 / 3,1 / 4,18 / 6,3 / 8) \\ 1(1 / 6) & 1(1 / 8) \\ 2(1 / 5,1 / 6) & 2(2 / 6) \\ 4(1 / 5,3 / 6) & 4(1 / 4,3 / 6) \\ 0 & 6(1 / 3,5 / 4) \\ 7(2 / 4,4 / 6,1 / 9) & 8(5 / 4,1 / 5,2 / 6) \\ & \end{array}$

Numbers following abbreviations of chemotherapy regimen denote cycle tempo in days

$A P$ apical periodontitis; $A B V D$ doxorubicin, bleomycin, vinblastine, dacarbazine; BEACOPP bleomycin, etoposide, doxorubicin, cyclophosphamide, vincristine, procarbazine, prednisone; $C H O E P$ cyclophosphamide, doxorubicin, vincristine, etoposide, prednisone; $C H O P$ cyclophosphamide, doxorubicin, vincristine, prednisone; COP cyclophosphamide, vincristine, prednisone; GA101 obinutuzumab; Hyper-CVAD hyperfractionated cyclophosphamide, vincristine, doxorubicin, dexamethasone, methotrexate, cytarabine; ICE ifosfamide, carboplatin, etoposide; $R$ rituximab; $M C L 2$ augmented RCHOP alternating with R-cytarabine; $C O E P$ cyclophosphamide, vincristine, etoposide, prednisone; $C P$ cyclophosphamide, prednisone

Data on exacerbation and local signs of infection, defined as registration of pain, and clinical signs of swelling or painful response to biting and/or palpation related to a tooth with AP were retrieved from the patients' records. In addition, data on exacerbation and local signs of infection emerging from periodontal tissues or partially erupted third molars were registered.

Medical data were retrieved from the medical records and consisted of hematological diagnosis and chemotherapy regimen. From the start of the first cycle of chemotherapy to the end of the observation period, administered antibiotics and the reason for antibiotic treatment, use of granulocyte colonystimulating factor (G-CSF), white blood cell counts (WBC), neutrophil counts, number of days with fever $\left(>38^{\circ} \mathrm{C}\right)$, number of ward admissions, number of admission days, and reason for admission to hospital during the observation period were registered. The patients' characteristics are presented in Table 1.

Statistical analyses of differences between groups were done using the Student's $t$ test or Fisher's exact test with the 
aid of the PRISM 6 software (GraphPad Software Inc., La Jolla, CA, USA). The level of significance was set at $P<0.05$.

The Regional Ethical Review Board in Gothenburg approved the study (no. 190-14), and the study was performed in accordance with the Declaration of Helsinki.

\section{Results}

For all the patients, the study period had an average duration of 4.4 months. Patients with AP were followed for an average of 4.5 months (median, 4.4; range, 1.8-9.5 months), and patients without AP were followed for an average of 4.3 months (median, 4.3; range, 2.3-7.4 months; Table 2). The mean WBC before the start of chemotherapy was $8.5 \times 10^{9} / \mathrm{L}$ for the study group and $8.6 \times 10^{9} / \mathrm{L}$ for the control group (Table 2). Data for two patients in the study group were missing. There were no significant differences in the WBC counts at treatment start between the group of patients with AP and the group without AP $(P=0.838)$ (Table 2$)$.

One or more teeth with AP were found in 86 of the 213 patients reviewed (Table 2). In total, 180 teeth with AP were registered among the 86 patients in the study group. Sixty-four teeth $(36 \%)$ had AP in teeth that were not previously root-filled, and 116 teeth (64\%) showed post-endodontic AP. Fifty patients had more than one tooth with AP, while 36 patients had only one tooth with AP. At the time of dental examination, the majority of the patients had started chemotherapy. Only 15 patients (of the 213 included patients) had undergone a dental examination prior to the start of chemotherapy.

Sixty-seven percent of patients in the study group had symptoms from the oral mucosa at baseline evaluation. Most frequently reported were dry mouth (58\%) and taste alterations $(41 \%)$. Oral ulceration was reported with a frequency of $5 \%$. Six percent $(n=5)$ of the patients reported dental tenderness. In the control group, symptoms from the oral mucosa were described in $75 \%$ of the patients. Most frequently reported were dry mouth $(60 \%)$ and taste alterations $(53 \%)$. Ulceration was reported with a frequency of $7 \%$. In the control group, $3 \%(n=$ 4 ) of the patients reported dental tenderness and $4 \%(n=$ 5) reported dentin hypersensitivity.

Seven patients $(8 \%)$ in the study group with AP experienced an exacerbation of their condition during the observation period (Table 3 ). Five of the exacerbations were associated with teeth that were not subjected to earlier root canal treatment, and two teeth showed incomplete root fillings (Table 3). None of the patients displayed facial swelling. Emergency dental treatment varied from extraction to trepanation, and permanent treatment ranged from extraction to root filling to apical surgery. Most of the patients with exacerbations were administered antibiotics, either as a prophylactic measure in connection with dental treatment or as part of emergency treatment (Table 3). Among the five patients in the study group that reported dental tenderness from teeth at baseline evaluation, three patients later experienced an exacerbation of the AP.

In five out of the seven patients, exacerbation occurred following the first cycle of chemotherapy (Table 3). The subtype of lymphoma diagnosis varied, as shown in Table 3. The radiographic images of the endodontic lesions causing the exacerbations did not show a distinct pattern regarding size, in either root canal-treated (Fig. 1a) or non-treated (Fig. 1b) teeth.

Of the patients with exacerbation, the WBC counts in six of the patients and neutrophil counts in two patients could be retrieved from the medical records, within 3 days of the date of exacerbation (Table 3 ). One patient had leukopenia (WBC $\left.<1 \times 10^{9} / \mathrm{L}\right)$ at the time of exacerbation. Three out of the seven patients were treated with G-CSF to shorten the period of neutropenia.

Table 2 Clinical data for patients with or without apical periodontitis

\begin{tabular}{|c|c|c|c|}
\hline & Apical periodontitis & No apical periodontitis & $\begin{array}{l}P \text { values for difference } \\
\text { between groups }\end{array}$ \\
\hline No. of patients & 86 & 127 & \\
\hline Study period in months: mean; median (range) & $4.5 ; 4.4(1.8-9.5)$ & $4.3 ; 4.4(2.3-7.4)$ & \\
\hline White blood cell counts: mean; median (range) ${ }^{\mathrm{a}}$ & $\begin{array}{l}8.5 \times 10^{9} / \mathrm{L} ; 7.15 \\
\quad(1.6-36.8)^{\mathrm{b}}\end{array}$ & $\begin{array}{c}8.6 \times 10^{9} / \mathrm{L} ; 7.6 \\
(0.2-57.8)\end{array}$ & $0.838^{\mathrm{c}}(n s)$ \\
\hline Patients with hospital admission because of fever/infection & 10 & 24 & $0.183^{\mathrm{d}}(n s)$ \\
\hline Hospital admission events & 18 & 32 & $0.512^{\mathrm{d}}(\mathrm{ns})$ \\
\hline Patients who received antibiotic treatment because of dental infection & 2 & 0 & $0.164^{\mathrm{d}}(n s)$ \\
\hline
\end{tabular}

The significance level is set to $p<0.05$

${ }^{a}$ At start of first cycle of chemotherapy

${ }^{\mathrm{b}}$ Missing data: two patients

${ }^{\mathrm{c}} P$ value for Student's $t$ test

${ }^{\mathrm{d}} P$ value for Fisher's exact test 


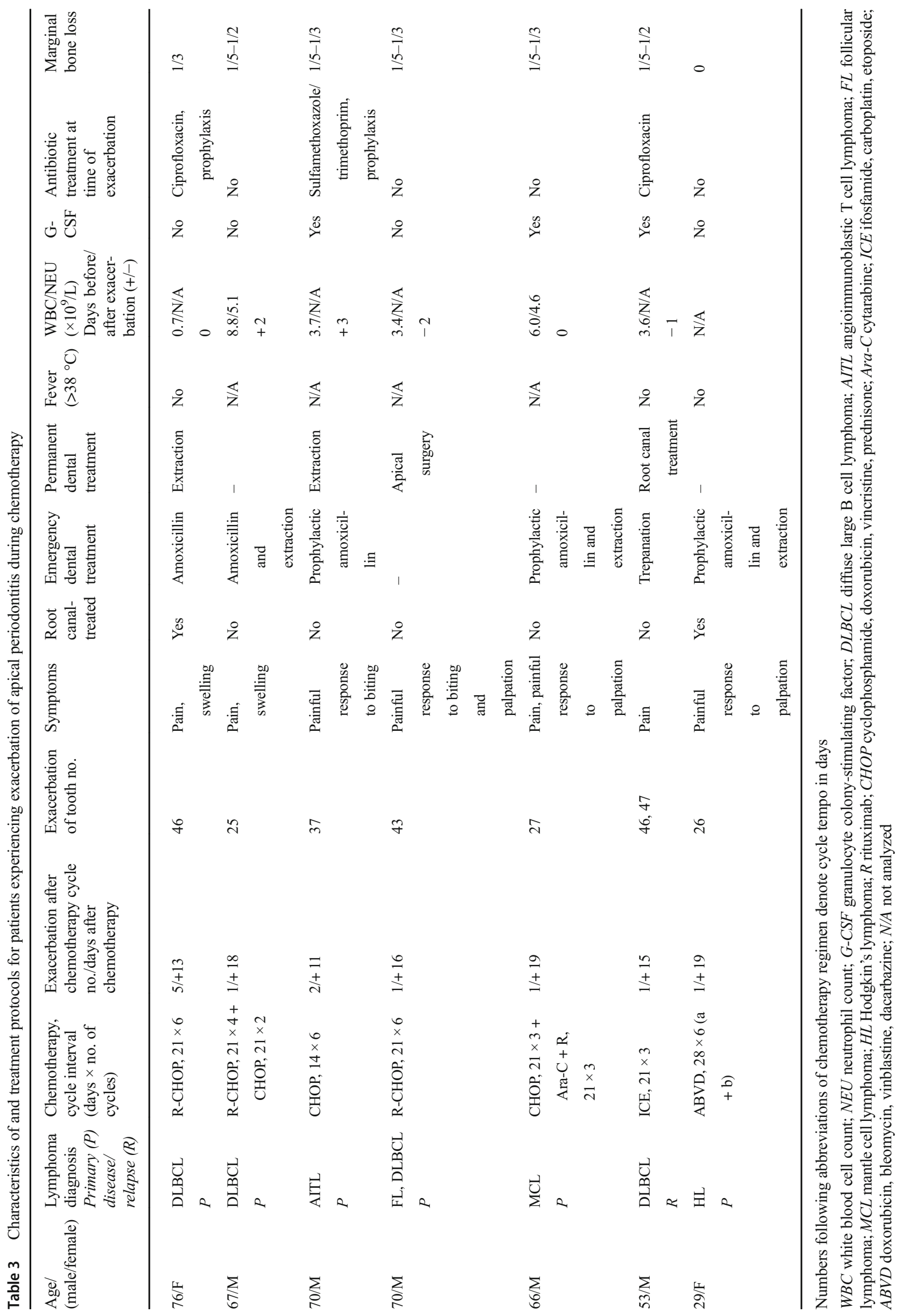


Fig. 1 Patient with diffuse large B cell lymphoma with a radiographic lesion around the mesial root of tooth 46 , diagnosed as symptomatic apical periodontitis after failed root canal treatment (a). Patient with follicular lymphoma and diffuse large B cell lymphoma with a radiographic lesion around tooth 43 , diagnosed as symptomatic apical periodontitis (b)
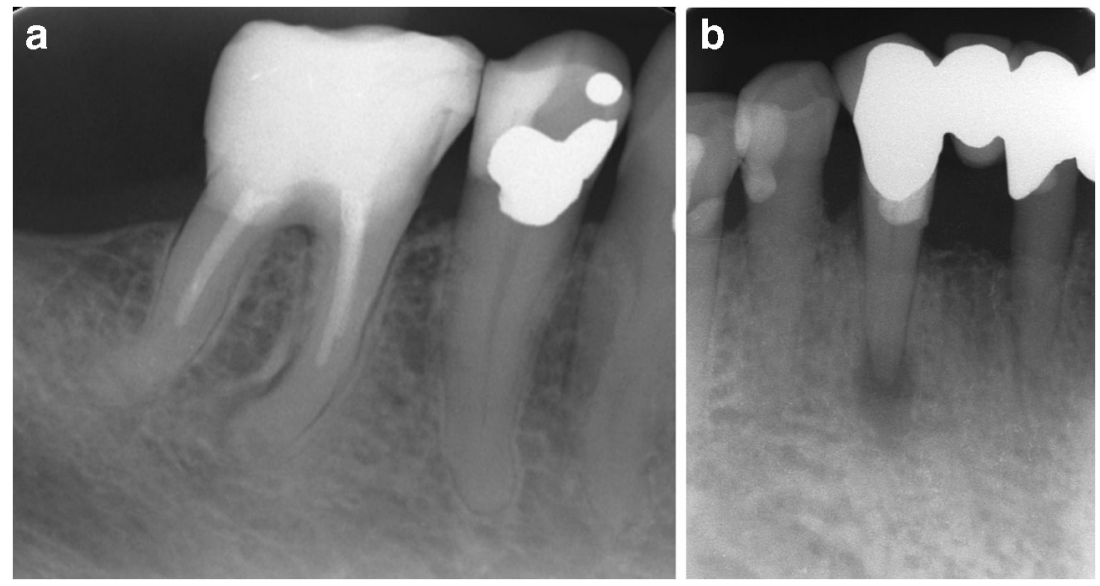

Comparing the study group with the control group, there was no significant difference $(P=0.183)$ in the number of patients with fever requiring hospital admission during chemotherapy (Table 2). No significant difference was noted between the two groups for hospital admission events due to fever or infection during chemotherapy $(P=0.512)$ or for antibiotic treatment owing to dental infections $(P=0.164)$ (Table 2).

In all, 38\% (33/86) of the patients in the study group and $20 \%(25 / 127)$ in the control group had a general marginal bone level loss equal to or greater than one-third of the root length. In the study group, 4\% (3/86) of the patients had partially erupted third molars, while the corresponding figure for the control group was 8\% (10/127). Exacerbation of chronic periodontal disease occurred in two patients during the observation period, according to the medical and dental records. No patient had registration of signs or symptoms of infection from partially erupted third molars.

\section{Discussion}

The present study shows that exacerbation of asymptomatic AP occurs during chemotherapy for lymphoma. However, no episode of exacerbation generated a significant impact on systemic health, such as fever or a need for hospital admission.

The risk for exacerbation of asymptomatic AP in a healthy population is not well known. Yu et al. have estimated the risk of exacerbation as $<6 \%$ over a 20 -year period [6]. Crosssectional studies have indicated an annual exacerbation incidence of $<5 \%$ [5].

In the present study, $8 \%$ of the patients with asymptomatic $\mathrm{AP}$ at baseline experienced an exacerbation during the observation period. Notably, five out of seven of those exacerbations occurred after the first cycle of chemotherapy.

The majority of the exacerbations were associated with teeth that had not been subjected to root canal treatment. This suggests a higher risk of exacerbation for untreated teeth with AP. Two patients experienced exacerbations in teeth that had AP in association with incomplete root fillings. It is well established that an incomplete root filling correlates with a remaining infection [3]. Necrotic pulp tissue and, in some cases, the periapical tissue harbor microorganisms that in an immunocompetent patient are usually controlled by the immune system. Immunosuppression due to chemotherapy reduces the number of leucocytes, leading to an increased risk for infection [1].

Of the 213 patients whose dental and medical records were reviewed in this study, $40 \%$ (86 patients) had at least one tooth with AP. This is in line with prevalence figures reported in epidemiologic surveys [12]. The observation period for patients with AP in the present study was in the range of 1.8 9.5 months, with an average of 4.5 months. The maximum observation period of 10 months indicates that the observed exacerbation frequency of $8 \%$ is higher than the estimated 5\% annual incidence reported previously [5]. Thus, chemotherapy seems to increase the risk that AP will become symptomatic, as compared to what may be expected in a healthy population.

Patients with severely deteriorated teeth received dental treatment despite the fact that chemotherapy had been started and they were excluded from the study. Thus, it is possible that our reported exacerbation frequency of AP would have been higher if patients with more severe dental disease had been included.

The lack of population-based studies on the risk for exacerbation of endodontic lesions hinders a statistical comparison of the exacerbation frequency reported in this study with the corresponding frequency in a healthy population. However, the exacerbation frequency reported here is much higher than the $<5 \%$ annual risk described in the earlier study [5].

Dental intervention in patients who are treated with chemotherapy due to cancer is a challenge for the dental community. The ideal situation is to complete the necessary dental treatment before chemotherapy is started. However, this is not always possible due to time limitations, the medical status of the patient, and urgency to initiate chemotherapy. 
Toljanic and colleagues have suggested in a prospective study that patients with chronic dental diseases without risk could proceed to chemotherapy without dental treatment [13]. In this study, patients diagnosed with acute dental pathology underwent dental treatment to remove the source of infection, but patients diagnosed with chronic dental pathology received no dental treatment [13]. They also reported that acute dental infections emerging during chemotherapy could be treated without medical complications [13].

In a recent prospective study, Schuurhuis and coworkers used the same protocol as described by Toljanic et al. whereby acute oral infections were eliminated prior to chemotherapy but chronic oral foci were left untreated in a group of patients with hematological malignancies [14]. Schuurhuis et al. found that the prevalence of exacerbation of chronic oral foci during hematologic treatment was $4 \%$, which is similar to the findings reported by Toljanic et al. [14]. The present study lends further support to the results presented in the two aforementioned studies.

Regarding analyses of blood samples (WBC, neutrophils), consistent data were missing. Interestingly, only one of the six patients for whom WBC counts were available at the time of exacerbation displayed leukopenia. A possible explanation for this is that some time elapsed between the onset of infection, occurrence of symptoms, and finally diagnosis and treatment. None of the patients in the exacerbation subgroup needed to be hospitalized or had a period of fever described in their medical record as a consequence of exacerbation. Thus, AP does not seem to lead to major medical consequences. This is probably due to the close monitoring of patients, whereby appropriate dental and medical treatments are started as soon as oral infections are diagnosed.

In similarity to AP, marginal periodontitis is a disease caused by infecting bacteria. In measuring the systemic effects of oral infections, the presence of periodontal disease is of importance. In patients with cancer, periodontal disease has been implicated as a major risk factor for septicemia [15]. Exacerbation of chronic periodontal disease occurred in two patients during the observation period, according to the medical and dental records. Since a full assessment of pocket depth is not performed routinely in our center for patients that have started chemotherapy, the level of periodontal disease is limited to an estimation of marginal bone loss, which is a limitation of the present study.

Partially erupted third molars (PEMs) are well known to cause infection around the partially erupted tooth [16]. Immunosuppression following high-dose chemotherapy increases the risk for local infection in the regions of partially erupted teeth [17]. In the present study, no local infection was registered for any of the patients with PEMs. Therefore, this type of oral infection did not influence the results.
Taken together, several sources with potential to cause oral infections were present in our group of patients. However, in the patients who developed exacerbations, acute infection was not attributable to causes other than AP.

In this study, no consistent patterns in relation to the subsets of lymphoma and chemotherapy regimens were seen in the patients with exacerbation of AP. Thus, despite the complexity of the subtypes of lymphoma and also of the treatment regimens, three features seem to predict exacerbation: (i) the tendency for exacerbation to occur after the first cycle of chemotherapy, (ii) the presence of a periapical lesion and absence of root filling, and (iii) incomplete root filling.

Although the dental infection-related events in this study were few and without severe medical consequences, all emerging infections in severely immunosuppressed individuals can lead to life-threatening conditions. According to Elad and coworkers, choosing the strategy of no dental treatment increases the probability to an additional 1.8 of every 1000 hematopoietic stem cell transplant patients dying secondary to dental infection [18]. Despite the findings in the present study and other studies $[13,14]$ that chronic dental infections do not pose a severe medical risk, this risk cannot be regarded as negligible. Importantly, the patients in the present study, as well as in the studies by Schuurhuis et al. [14] and Toljanic et al. [13], received dental treatment of acute oral infections.

In conclusion, $8 \%$ of patients with AP experienced an exacerbation that required dental treatment and/or antibiotic treatment in an outpatient setting. However, no patient with dental AP had complications that required hospital admission due to fever or infection. Prompt dental treatment in each case of exacerbation prevented more serious infection-related complications. Bearing in mind the time limitations and available resources, it is recommended to establish dental treatment protocols that are safe and efficacious for patients who are scheduled for intensive chemotherapy.

Acknowledgments The authors acknowledge the valuable contribution of Dr. Parissa Mokthari in reviewing the patients' records. This study was funded by grants from the Local Research and Development Board for Gothenburg and Södra Bohuslän, Region Västra Götaland, Sweden (grant nos. VGFOUGSB-394111 and VGFOUGSB-394241).

Funding This study was funded by grants from the Local Research and Development Board for Gothenburg and Södra Bohuslän, Region Västra Götaland, Sweden (VGFOUGSB-394111, VGFOUGSB-394241).

\section{Compliance with ethical standards}

Conflict of interest The authors declare that they have no conflicts of interest.

Ethical approval All procedures performed in the studies involving human participants were in accordance with the ethical standards of the institutional and/or national research committee and with the 1964 
Helsinki Declaration and its later amendments or comparable ethical standards. The Regional Ethical Review Board in Gothenburg approved the study (no. 190-14).

Informed consent For this type of study, formal consent is not required.

Open Access This article is distributed under the terms of the Creative Commons Attribution 4.0 International License (http:// creativecommons.org/licenses/by/4.0/), which permits unrestricted use, distribution, and reproduction in any medium, provided you give appropriate credit to the original author(s) and the source, provide a link to the Creative Commons license, and indicate if changes were made.

\section{References}

1. Vento S, Cainelli F (2003) Infections in patients with cancer undergoing chemotherapy: aetiology, prevention, and treatment. Lancet Oncol 4(10):595-604

2. Nair PN (2004) Pathogenesis of apical periodontitis and the causes of endodontic failures. Crit Rev Oral Biol Med 15(6):348-381

3. Frisk F, Hugoson A, Hakeberg M (2008) Technical quality of root fillings and periapical status in root filled teeth in Jonkoping, Sweden. Int Endod J 41(11):958-968. https://doi.org/10.1111/j. 1365-2591.2008.01457.x

4. Siqueira JF Jr, Rocas IN (2013) Microbiology and treatment of acute apical abscesses. Clin Microbiol Rev 26(2):255-273. https://doi.org/10.1128/cmr.00082-12

5. Eriksen HM (2008) Epidemiology of apical periodontitits. In: Ørstavik D, Pitt Ford T (eds) Essential endodontology, 2nd edn. Blackwell Munksgaard Ltd, Frederiksberg, p 267

6. Yu VS, Messer HH, Yee R, Shen L (2012) Incidence and impact of painful exacerbations in a cohort with post-treatment persistent endodontic lesions. J Endod 38(1):41-46. https://doi.org/10.1016/j. joen.2011.10.006

7. Hong CHL, Hu S, Haverman T, Stokman M, Napenas JJ, Braber JB, Gerber E, Geuke M, Vardas E, Waltimo T, Jensen SB, Saunders DP (2018) A systematic review of dental disease management in cancer patients. Support Care Cancer 26(1):155-174. https://doi. org/10.1007/s00520-017-3829-y

8. Freifeld AG, Bow EJ, Sepkowitz KA, Boeckh MJ, Ito JI, Mullen CA, Raad II, Rolston KV, Young JA, Wingard JR, Infectious Diseases Society of A (2011) Clinical practice guideline for the use of antimicrobial agents in neutropenic patients with cancer: 2010 update by the Infectious Diseases Society of America. Clin Infect Dis 52(4):e56-e93. https://doi.org/10.1093/cid/cir073

9. Raber-Durlacher JE, Barasch A, Peterson DE, Lalla RV, Schubert MM, Fibbe WE (2004) Oral complications and management considerations in patients treated with high-dose chemotherapy. Support Cancer Ther 1(4):219-229. https://doi.org/10.3816/SCT. 2004.n.014
10. Brennan MT, Woo SB, Lockhart PB (2008) Dental treatment planning and management in the patient who has cancer. Dent Clin N Am 52(1):19-37, vii. https://doi.org/10.1016/j.cden.2007.10.003

11. Elad S, Raber-Durlacher JE, Brennan MT, Saunders DP, Mank AP, Zadik Y, Quinn B, Epstein JB, Blijlevens NMA, Waltimo T, Passweg JR, Correa MEP, Dahllöf G, Garming-Legert KUE, Logan RM, Potting CMJ, Shapira MY, Soga Y, Stringer J, Stokman MA, Vokurka S, Wallhult E, Yarom N, Jensen SB (2015) Basic oral care for hematology-oncology patients and hematopoietic stem cell transplantation recipients: a position paper from the joint task force of the Multinational Association of Supportive Care in Cancer/International Society of Oral Oncology (MASCC/ISOO) and the European Society for Blood and Marrow Transplantation (EBMT). Support Care Cancer 23(1):223-236. https://doi.org/10.1007/s00520-014-2378-x

12. Frisk F, Hakeberg M (2005) A 24-year follow-up of root filled teeth and periapical health amongst middle aged and elderly women in Goteborg, Sweden. Int Endod J 38(4):246-254. https://doi.org/10. 1111/j.1365-2591.2005.00944.x

13. Toljanic JA, Bedard JF, Larson RA, Fox JP (1999) A prospective pilot study to evaluate a new dental assessment and treatment paradigm for patients scheduled to undergo intensive chemotherapy for cancer. Cancer 85(8):1843-1848

14. Schuurhuis JM, Span LF, Stokman MA, van Winkelhoff AJ, Vissink A, Spijkervet FK (2016) Effect of leaving chronic oral foci untreated on infectious complications during intensive chemotherapy. Br J Cancer 114(9):972-978. https://doi.org/10.1038/bjc.2016. 60

15. Raber-Durlacher JE, Epstein JB, Raber J, van Dissel JT, van Winkelhoff AJ, Guiot HF, van der Velden U (2002) Periodontal infection in cancer patients treated with high-dose chemotherapy. Support Care Cancer 10(6):466-473. https://doi.org/10.1007/ s00520-002-0346-3

16. von Wowern N, Nielsen HO (1989) The fate of impacted lower third molars after the age of 20. A four-year clinical follow-up. Int J Oral Maxillofac Surg 18(5):277-280

17. Ohman D, Bjork Y, Bratel J, Kristiansson C, Johansson P, Johansson JE, Brune M, Hasseus B (2010) Partially erupted third molars as a potential source of infection in patients receiving peripheral stem cell transplantation for malignant diseases: a retrospective study. Eur J Oral Sci 118(1):53-58. https://doi.org/10. 1111/j.1600-0722.2009.00705.x

18. Elad S, Thierer T, Bitan M, Shapira MY, Meyerowitz C (2008) A decision analysis: the dental management of patients prior to hematology cytotoxic therapy or hematopoietic stem cell transplantation. Oral Oncol 44(1):37-42. https://doi.org/10.1016/j.oraloncology. 2006.12.006

Publisher's note Springer Nature remains neutral with regard to jurisdictional claims in published maps and institutional affiliations. 